\title{
Metabarcoding gastrointestinal nematodes in sympatric endemic and non-endemic species in Ranomafana National Park, Madagascar
}

\section{Aivelo, Tuomas Juho Eero}

2018-02

Aivelo , T J E , Medlar , A J , Löytynoja , A P , Laakkonen , J T \& Jernvall , J T 2018 , ' Metabarcoding gastrointestinal nematodes in sympatric endemic and non-endemic species in Ranomafana National Park, Madagascar ' , International Journal of Primatology , vol. 39 , pÿno. 1 , pp. 4964 . https://doi.org/10.1007/s10764-017-0010-x

http://hdl.handle.net/10138/311338

https://doi.org/10.1007/s10764-017-0010-x

acceptedVersion

Downloaded from Helda, University of Helsinki institutional repository.

This is an electronic reprint of the original article.

This reprint may differ from the original in pagination and typographic detail.

Please cite the original version. 


\section{Metabarcoding gastrointestinal nematodes in sympatric}

2

3

4

5 Tuomas Aivelo ${ }^{1,2,3}$, Alan Medlar ${ }^{1}$, Ari Löytynoja ${ }^{1}$, Juha Laakkonen ${ }^{4}$, Jukka Jernvall ${ }^{1}$

$6 \quad{ }^{1}$ Institute of Biotechnology, University of Helsinki, 00014 Helsinki, Finland

$7 \quad{ }^{2}$ Department of Biosciences, University of Helsinki, 00014 Helsinki, Finland

$8{ }^{3}$ Department of Evolutionary Biology and Environmental Studies, University of Zürich, CH-

98057 Zürich, Switzerland

$10{ }^{4}$ Department of Veterinary Biosciences, University of Helsinki, 00014 Helsinki, Finland 


\section{Abstract}

While sympatric species are known to host the same parasites species, surveys contrasting parasite assemblages between sympatric species are rare. To understand how parasite assemblages between sympatric host species differ in a given locality, we used a non-invasive identification method based on high-throughput sequencing. We collected fecal samples from mouse lemurs and sympatric species in Ranomafana National Park, Madagascar, during 20102012 and identified their parasites by metabarcoding; sequencing the small ribosomal subunit (18S) gene. Our survey included 11 host species, including: endemic primates, rodents, frogs, gastropods and non-endemic black rats and dogs. We identified nine putative species of parasites between host species, although their correspondence to actual parasite species is not clear as the resolution of the marker gene differs between nematode clades. For the host species that were successfully sampled with ten or more positive occurrences of nematodes, i.e., mouse lemurs, black rats and frogs, the parasite assemblanges differed significantly between host species, sampling sites and sampling years. Our metabarcoding method shows promise in interrogating parasite assemblages in sympatric host species and emphasizes the importance of choosing marker regions for parasite identification accuracy.

Keywords: Lemurs, Metabarcoding, Parasites, Invasive species, Non-invasive sampling

31 Running title: Metabarcoding parasite assemblages in sympatric host species 


\section{Introduction}

Parasite dynamics research is hindered by parasite groups that are difficult to identify; requiring extensive taxonomical expertise. Furthermore, the identification of intestinal nematode species traditionally requires dissection of host animals to collect and morphologically identify adult nematode specimens. This approach is time-consuming and due to its' invasiveness, is not always feasible.

The standard method for assessing gastrointestinal parasites non-invasively is fecal analysis (Gillespie 2006). When identification is based on egg or larval morphology, this often leads to parasite identification at high taxonomical levels, such as order or family, and rarely allows for identification at the genera or species-level. Several procedures based on molecular markers have been proposed for non-invasive assessment of parasitic nematodes (e.g.: Wimmer et al. 2004). Although these can reliably identify specific species or strains, the published procedures lack the broad spectrum needed for host populations of unknown parasite communities. Barcoding, i.e., identifying species by sequencing a marker gene, is the method of choice to identify high diversity among nematode communities. Furthermore, highthroughput sequencing allows for the identification of several nematode taxons from a single fecal sample, i.e., metabarcoding (Aivelo and Medlar 2017; Taberlet et al. 2012), but few studies have used this method to identify gastrointestinal nematodes (Avramenko et al. 2015; Lott et al. 2015; Tanaka et al. 2014). As species are not defined by sequence, the groupings resulting from barcoding analyses are referred as operational taxonomic units (OTUs) (Blaxter et al. 2005). OTUs may not correspond to actual species but to taxons of lower or 4 higher level (Bik et al. 2012). 
While parasite communities in sympatric primates have already been studied (e.g., Kouassi et al. 2015; Loudon and Sauther 2013; Maldonado-López et al. 2014; Muriuki et al. 1998; Petrášová et al. 2010; Pourrut et al. 2011; Schwensow et al. 2010; Teichroeb et al. 2009; Trejo-Macías et al. 2007; Trejo-Macías and Estrada 2012), there have been relatively few studies comparing primate parasite species composition to sympatric non-primate mammals. Nevertheless, parasite sharing appears to be common in wild mammals (Chakraborty et al. 2015; Dallas and Presley 2014; Kouassi et al. 2015). Parasite communities can also be affected by the introduction of non-endemic host species that provide new competent hosts for endemic parasites (Dunn et al. 2012; Kelly et al. 2009) or they can bring new parasite species to the ecosystem (Hudson and Greenman 1998; Taraschewski 2006). Introduced hosts tend to have lower parasite species diversity than in their endemic area (Dobson and May 1986; Freeland 1983; Torchin et al. 2003), which may be due to loss of their original parasites during colonization (MacLeod et al. 2010).

To our knowledge, there have been no studies on metabarcoding intestinal parasites from different sympatric host species. Our principal aim was to assess whether metabarcoding is a viable tool for such parasitological surveys. We explored gastrointestinal nematode assemblages in several species living within or in the peripheral zone of Ranomafana National Park, Madagascar. Using invasive black rats, we also tested if molecular identifications from larvae acquired from fecal samples matched morphological identifications made from adult nematodes. Ranomafana National Park is a suitable ecosystem for study, as it has high biodiversity, including 13 primate species, with notable anthropogenic disturbance and contains several non-endemic mammalian species. In Ranomafana National Park, a number of endemic species are threatened with extinction, including critically endangered golden and 
greater bamboo lemurs (Hapalemur aureus (Andriaholinirina et al. 2014a) and Prolemur simus (Andriaholinirina et al. 2014b), respectively). We hope that our method could help conservation efforts and facilitate wildlife health assessment within biodiversity hotspots. The research questions were: i) how well the $18 \mathrm{~S}$ marker gene can be used to survey intestinal parasite assemblages and ii) do non-endemic and endemic host species have similar nematode assemblages. We expected similar parasite assemblages between closely related species and between species sharing the same ecological niches, i.e., terrestrial species would have more overlap with each other compared to arboreal species.

\section{Methods}

\section{Sampling}

We collected fecal samples (Table 1) from sympatric species from September to December in 2010, 2011 and 2012 in southeastern Madagascar (21 ${ }^{\circ} 16^{\prime}$ S latitude and 47 $20^{\prime}$ E longitude). The national park is established on lowland to montane rainforest between 500 and 1500 meters elevation. The park consists of 43500 hectares of protected area as well as a peripheral zone with limited protection (Wright and Andriamihaja 2002). We collected mouse lemur samples nightly from two different transects, the first one within the National Park and the second on the periphery of the park in Centre Valbio's campsite. We laid 50 live traps $(22.2 \mathrm{x}$ 6.6 x $6.6 \mathrm{~cm}$; XLK, Sherman Traps Inc., Florida USA) along a trail at 50 meter intervals, an hour before sunset. Black rats (Rattus rattus), snails (Gastropoda sp.) and endemic rodents (Nesomys audeberti and Eliurus spp.) were also caught as a side catch in the same traps. We additionally used these two transects for opportunistic sampling of medium-sized lemurs 
104 (Eulemur rubriventer, Hapalemur aureus, Prolemur simus), domesticated dogs which range

105 freely within the local village and forested areas (Canis lupus) and frogs (Ptychadena spp.

106 and Mantidactylus spp.). We collected black rat samples from an additional location on the

107 peripheral zone of the park near Ambatovory. All sites contained secondary forest growth with

108 endemic and non-endemic trees.

109

110 We collected the traps three hours after sunset, sampled feces from the traps and brought any

111 captured black rats and mouse lemurs to the laboratory of Centre Valbio. We washed the traps

112 after each use and dried them in sunlight to decrease the chance of contamination from

113 previous captures. We terminated invasive black rat specimens and examined a subset $(\mathrm{n}=17)$

114 for adult nematodes in the gastrointestinal tract. We dissected the rats, opened their

115 gastrointestinal tract from stomach to anus, observed the gut lining and contents under a

116 microscope in saline solution and collected all helminths.

118 Ethical note

119 We minimized the duration that animals were kept in captivity, especially during the mouse

120 lemur mating season. We released the mouse lemurs as soon as we had collected the data from

121 the individual. We identified and released other captures on-site. We handled mouse lemurs

122 under red light to minimize stress. The procedures used were consistent with ethical standards

123 and approved by the trilateral commission (CAFF/CORE) in Madagascar (permits:

124 203/11/MEF/SG/DGF/DCB.SAP/SCBSE and 203/12/MEF/SG/DGF/DCB.SAP/SCBSE)

125

126 DNA isolation and sequencing

127 After collecting the fecal matter, we used Baermann's method to isolate the nematodes 
128 (Baermann 1917). We placed the fecal matter on a tissue (one half of 1-ply Kimwipe,

129 Kimberly-Clark Europe Ltd., Surrey, United Kingdom), folded the tissue and tied it with

130 string. We then placed this packet on a sterile glass funnel which was filled with

131 approximately $37^{\circ} \mathrm{C}$ distilled water. This allows all the living nematode larvae to swim out of

132 the fecal matter into the water. We collected the samples two days later, centrifuged them for 5

133 minutes at $2800 \mathrm{rcf}$ and discarded the supernatant. We quantified the number of nematode

134 larvae by examining the pellet under the microscope and stored the larvae in $70 \%$ ethanol in a

135 freezer at $-18^{\circ} \mathrm{C}$. It should be noted that Baermann's method only isolates nematodes which

136 have a free-living stage and thus we, therefore, could not acquire entire nematode

137 communities. We refer to the partially resolved parasite communities as assemblages. We

138 tested approximately every fifth rat fecal sample $(\mathrm{n}=18)$ after Baermann extraction by visual

139 screening on flotation liquid and did not find any residual nematode parasites.

140

141 For nematode DNA extraction, we used half of the visible larvae mass; approximately 40

142 microliters of liquid. For DNA extraction, we centrifuged the sample and removed any

143 ethanol. For adult nematodes collected directly from dissected rat intestine, we used one

144 individual or a part of an individual. The sample was incubated for 2 hours at room

145 temperature in milliQ water to rehydrate the nematodes and remove excess ethanol. To lyse

146 the cells, we centrifuged the sample, removed the water and incubated the sample in 400

147 microliters of TE buffer (10 mM Tris-HCl, $1 \mathrm{mM}$ EDTA, $\mathrm{pH}$ 8.5) together with 40

148 micrograms of proteinase $\mathrm{K}$ overnight at $56^{\circ} \mathrm{C}$. We collected DNA with isopropanol

149 precipitation: we centrifuged the samples for 10 minutes at $15000 \mathrm{rcf}$ and discarded the pellet.

150 We mixed the supernatant with 400 microliters of isopropanol and incubated for 5 minutes at

151 room temperature. After precipitation we centrifuged the samples for 10 minutes at $15000 \mathrm{rcf}$, 
152 discarded the supernatant and washed the resultant pellet twice using 500 microliters of $70 \%$

$153(\mathrm{v} / \mathrm{v})$ ethanol. After ensuring that all ethanol had evaporated, we suspended the sample in 30

154 microliters of TE buffer.

155

156 To amplify the ribosomal small subunit gene (18S) we used primers from Bhadury and Austen

157 (2010): M18F: 5'-AGRGGTGAAATYCGTGGAC-3' and M18R: 5'-

158 TCTCGCTCGTTATCGGAAT-3'. These primers were designed for marine nematodes with

159 high-specificity and minimal co-interference from other eukaryotes. The PCR mix included 1

160 unit Phusion high-fidelity polymerase (Thermo Fisher Scientific, Inc, Waltham, MA, USA)

161 with buffer, 10-100 ng (0.5-5.0 microliter) of template, $0.2 \mu \mathrm{M}$ primers, $200 \mu \mathrm{M}$ dNTP mix,

$1621.5 \mathrm{mM} \mathrm{MgCl} 2$ and 2\% DMSO per reaction. The PCR program included initial denaturation

163 at $98^{\circ} \mathrm{C}$ for two minutes, then $30-40$ cycles of $15 \mathrm{~s}$ denaturation at $98^{\circ} \mathrm{C}$, annealing at $53^{\circ} \mathrm{C}$ for

16430 s and 30 s extension at $72^{\circ} \mathrm{C}$ and ending with 10 minutes of final elongation at $72^{\circ} \mathrm{C}$. PCR

165 results were checked on a $1 \%$ agarose gel.

166

167 Amplicons were sequenced at the DNA sequencing and Genomic laboratory, Institute of

168 Biotechnology, University of Helsinki using a Roche 454 Genome Sequencer FLX+.

169

170 Sequence analysis

171 We performed data analysis using the Séance pipeline for reference-based phylogenetic

172 amplicon analysis (Medlar et al. 2014). We used Ampliconnoise (ver. 1.29) (Quince et al.

173 2011) to denoise each sample. We discarded sequences with ambiguous base calls, more than

1741 error in the multiplexing barcode or more than two errors in the primer sequence, removed

175 multiplexing barcodes and primers and truncated all sequences to $250 \mathrm{bp}$. We removed 
176 putative chimeric sequences using UCHIME (ver. 4.2.40) in de novo mode (Edgar et al. 2011)

177 and excluded all sequences with a copy number less than 5. We expect that a majority of the

178 sequences filtered out will represent PCR artefacts and sequencing errors not caught during

179 preprocessing. We performed the clustering of the sequences with a similarity threshold of

$18099 \%$. Séance's clustering methodology explicitly models homopolymer length uncertainty in

181454 data across many samples.

182

183 Clusters were labelled using Séance's taxonomical labelling strategy. In brief, each cluster is

184 formed around a (generally highly abundant) centroid sequence, which we use to perform a

185 MegaBLAST (Camacho et al. 2009) search of the NR (non-redundant) database at NCBI. We

186 excluded results with lower than $90 \%$ identity and those from environmental and

187 metagenomic samples. As each sequence is only $250 \mathrm{bp}$ long there is often some ambiguity as

188 to which species it is most similar to, so instead we report the lowest common ancestor from

189 the NCBI taxonomy of all top scoring BLAST hits (i.e., the taxon which contains all the

190 taxons representing the top hits). For comparison, we generated labels using the same

191 procedure, but substituting the NR database with SILVA (SSURef NR ver. 115) (Quast et al.

192 2013), which contains its own taxonomic data.

194 Séance uses a phylogenetic placement strategy for phylogenetic analysis. For this we need a

195 reference tree to extend with the cluster sequences. To build the reference tree, we extracted

196 the complete 18S rRNA gene sequence from all 1320 members of the phylum Nematoda

197 found in SILVA ver. 115 and built a tree with RAxML (ver. 7.2.8) (Stamatakis 2006). RAxML

198 was run with the GTR+gamma substitution model for 10 repetitions. We used Séance's

199 phylogenetic placement command to place the cluster centroid sequences into the reference 
tree. Visualizations were also produced with Séance.

\section{Putative species determination}

203 As the resulting OTUs may include non-nematode, contamination, and spurious OTUs caused

204 by amplification or sequencing errors, we extracted what we termed putative species from the 205 results of each cluster analysis. To generate a putative list of nematode species, we first removed all clusters with taxonomic labels to phyla other than Nematoda. To ensure that we report only nematodes parasitic to the host species we sampled, we studied OTU cooccurrence patterns, e.g., known dipteran parasitizing nematode clusters were removed as

209 they were only found in samples together with dipteran clusters. Free-living nematodes may

210 have contaminated our samples, for example, by attaching to a rodents' foot and then 211 transferring to the feces prior to collection. OTUs were deemed to be contamination from soil

212 nematodes when the best hits for clusters were soil nematode groups and there was a

213 reasonable chance of contamination. Finally, it is likely that there is a number of spurious

214 OTUs due to amplification and sequencing errors. To conservatively take these into account, 215 we merged OTUs that formed a homogenous group. The criteria for merging was that a) the 216 OTUs were clustered to the same taxon, b) they formed a monophyletic group in the 217 phylogenetic tree, c) there was one clearly dominant OTU in this group and d) the OTUs 218 occurred in the same individuals (so-called head-tail structure (Porazinska, Giblin-Davis, 219 Sung, et al. 2010; Figure S1). Nevertheless, it should be noted that these putative species can 220 contain more than one parasite species or, theoretically, they can also reveal cryptic species of 221 parasites, i.e., one parasite species can be divided into two or more putative species.

\section{Data availability}


224 The raw sequences have been deposited in the Sequence Read Archive under SRA number

225 SRP042187. The metadata for the samples, including the matching of samples to sample

226 accession numbers can be found in the data file in Figshare: doi:

227 10.6084/m9.figshare.1289310

Statistical analysis

230 We performed all statistical tests and their visualizations in $\mathrm{R}$ using the stats package (R Core

231 Team 2013) and the mvabund package (Wang et al. 2016).

232 To assess the resolving ability of the particular primers we used, we extracted all nematode

$23318 \mathrm{~S}$ sequences from the SILVA database, extracted the marker region using the primers,

234 trimmed the sequences to $250 \mathrm{bp}$ and clustered them at $99 \%$ similarity. Then we assigned

235 labels for each of the clusters and quantified the number of unique clusters (i.e., clusters

236 composed of different sets of sequences) within each taxon corresponding to our observed

237 putative species labels.

239 We calculated parasite prevalence for host taxa with 10 or more samples and analyzed

240 parasite assemblages in host taxa with 10 or more successful sequencings (mouse lemurs,

241 black rats and frogs) by using a generalized linear model with a binomial link function and

242 using trapping site and year as variables in addition to host species. As we were not able to

243 identify putative species in all positive samples (i.e., samples without successful sequencing),

244 we removed a similar proportion of negative samples from the analysis. P-values are assigned

245 by resampling which bootstraps probability integral transform residuals.

\section{Results}


249 We collected a total of 872 samples, of which 571 contained nematodes and 249 were

250 successfully sequenced (Table 1). We dissected 17 black rats of which 14 were positive for

251 nematodes in the gastrointestinal tract. The fecal samples of these 14 rats were also positive

252 for nematodes. The remaining three rats were all correspondingly negative based on their

253 fecal samples. There were two distinct morphotypes of nematodes: in the first two thirds of

254 the small intestine we collected nematodes resembling Nippostrongylus $\mathrm{sp} .(\mathrm{n}=14)$ and from

255 the stomach, nematodes that resembled Mastophorus sp. $(\mathrm{n}=2)$.

257 Sequencing and sequence analysis

258 The amplification and sequencing success rates were variable, ranging from $100 \%$ success in 259 gastropods to $0 \%$ in Eliurus and Nesomys spp. (Table 1). If amplification did not succeed on

260 the first try, we attempted reamplification. If amplification was still unsuccessful, we

261 reisolated the DNA and amplified it again. For the larval samples of mouse lemurs, there was

262 approximately $30 \%$ success in the first isolation and $22 \%$ success on the second isolation.

264 Table 1: Number of collected samples from study species in Ranomafana National Park, Madagascar between

265 September 2010 and December 2012, and species information regarding if species are arboreal (A) or terrestrial

266 (T), nocturnal (N), cathemeral (C) or diurnal (D), omnivores (O) or herbivores (H) (Nowak 1999a, 1999b) and

267 their sample counts and sequencing successes.

\begin{tabular}{ccccccc} 
Nic & Acti & Feedi & Total & Positive for & Successful & Nematode \\
he & vity & ng & number of & nematodes & sequencing & prevalence \\
& & \multicolumn{3}{c}{ the samples } & & $(\%)$
\end{tabular}

\begin{tabular}{lllllllll}
\hline Rufous mouse lemur & Microcebus rufus & A & N & O & 632 & 469 & 212 & 74 \\
Red-bellied brown & Eulemur & A & C & H & 7 & 3 & 1 &
\end{tabular}


lemur

Golden bamboo

lemur

Greater bamboo

lemur

Tufted-tailed rats

Eliurus spp.

Nesomys spp.

Dog

Frogs

Snails

Hapalemur

aureus

Black rat
Prolemur simus

A

D $\quad \mathrm{H}$

$\begin{array}{llll}\text { A, } & \mathrm{N} & \mathrm{O} & 82\end{array}$

3

0

4
Rattus rattus

- dissections

Canis lupus

T C

T N

Ranoidea

C H

$\mathrm{O}$

68

17

O 5

O 40

Gastropoda sp.

$\begin{array}{llll}\mathrm{T} & \mathrm{C} & \mathrm{H} & 4\end{array}$

20

12

268

269 We had a total of 677,451 reads from 290 samples. After preprocessing we had 409,088 high

270 quality reads, which were comprised of 7,308 unique sequences. The median number of high

271 quality reads per sample was 722 with an inter-quartile range of 279-2098. When all

272 sequences with copy number less than 5 were removed, we had a total of 308 unique

273 sequences, which is representative of $97.3 \%$ of the reads that passed quality control. We

274 performed sequence clustering with a similarity threshold of 99\%, which resulted in 35

275 OTUs. Of these OTUs 16 had a taxonomic label other than Nematoda. Most of the

276 contamination was most likely due to dipterans laying eggs in the samples during processing.

277 One OTU co-occurred only with dipteran contamination and was labelled as Howardula sp., a

278 nematode species parasitic in flies. This OTU was therefore classified as contamination.

279 Furthermore, there were 3 OTUs labelled as soil nematodes and recovered only with contact

280 to the soil. There were also matches to the soil nematodes in samples directly collected from 
rodents without contact to the soil and these were deemed to be parasitic nematodes ( 2 OTUs).

After processing, we had 9 putative species (Table 2; Figure 1).

\section{Resolution and reliability of putative nematode species}

The putative nematode species were named using the lowest common ancestor in the NCBI taxonomy for all top scoring BLAST hits for the centroid sequence. With the exception of PS3 and PS4, the putative nematode species were labelled to the genus level. However, some of these matches were free-living nematode genera, like PS2 (Caenorhabditis) and PS6 (Panagrellus) (Table 2). We performed labelling also with a curated database (SILVA) and the results were concordant, but more conservative than with NCBI NR (Table 2). After quality

294 control and curation, we had a total of 254 samples which included parasitic nematodes (Table 295 1). The resolving ability of the primers differs substantially between putative species labels (Table 2): e.g., the clusters labelled Chromadorea could consist of 2 to 103 species, while two Rhabditoides spp. (which belong to Chromadorea) clusters have only one described species in 298 them.

300 To assess the reliability of using Baermann's method, i.e., larvae developed from the fecal 301 samples, as a proxy for which adult specimens are present in the gastrointestinal tract, we compared the putative species from the dissected host black rat individuals in which we got

303 successful sequencing from both larval and intestinal samples. Nippostrongylus-like adult

304 specimens and the majority of the corresponding larval amplicons belonged to PS3

305 (Strongylida): two of the larval samples corresponded to their respective adult intestinal 
306 nematodes, but one larval sample did not contain the expected PS3 but rather PS1

307 (Strongyloides). The nematodes identified as Mastophorus sp. did not occur in larval samples

308 though it amplified well from the two adult gastrointestinal samples.

309

310 Table 2: Putative species and their potential taxonomic labels from study host species in Ranomafana National

311 Park, Madagascar between September 2010 and December 2012. There is a wide difference between the

312 taxonomic resolution of the lowest common ancestor of the top scoring BLAST hits in NR and SILVA database.

313 Some species are resolved to genera level (like Strongyloides and Syphacia), while other samples are resolved to

314 much higher taxa (like Chromadorea and Strongylida). The SILVA database gives more conservative labels. The

315 next two columns include the closest BLAST match for the centroid sequence and other close BLAST matches.

316 The rightmost column gives the number of unique clusters resulting from clustering all samples contained in

317 SILVA database in a lowest common ancestor taxon and total number of sequences with the taxon.

\begin{tabular}{|c|c|c|c|c|c|}
\hline $\begin{array}{l}\text { Putative } \\
\text { species }\end{array}$ & $\begin{array}{l}\text { LCA from NR } \\
\text { database }\end{array}$ & $\begin{array}{l}\text { LCA from SILVA } \\
\text { database }\end{array}$ & $\begin{array}{l}\text { Centroid BLAST } \\
\text { match }\end{array}$ & $\begin{array}{l}\text { Other close BLAST } \\
\text { matches }\end{array}$ & $\begin{array}{l}\text { Unique clusters / } \\
\text { sequences in SILVA }\end{array}$ \\
\hline 1 & Strongyloides & Strongyloides & S. stercoralis & S. procyonis & $3 / 10$ \\
\hline 2 & Caenorhabditis & Caenorhabditis & C. elegans & $\begin{array}{l}\text { several Caenorhabditis } \\
\text { spp. }\end{array}$ & $3 / 3$ \\
\hline 3 & Strongylida & Rhabditidae & $\begin{array}{l}\text { Gurltia paralysans / } \\
\text { Dictyocaulus }\end{array}$ & $\begin{array}{l}\text { Strongylus, Filaroides, } \\
\text { Trichostrongylus, } \\
\text { Ancylostoma, } \\
\text { Angiostrongylus }\end{array}$ & $53 / 135$ \\
\hline 4 & Chromadorea & Chromadorea & $\begin{array}{l}\text { Physaloptera } \\
\text { thalacomys }\end{array}$ & $\begin{array}{l}\text { Gongylonema } \\
\text { pulchrum }\end{array}$ & $147 / 291$ \\
\hline 5 & Enterobius & Enterobius & E. vermicularis & & $1 / 1$ \\
\hline 6 & Panagrellus & Panagrellus & P. redividus & & $4 / 8$ \\
\hline 7 & Rhabditoides & Rhabditoides & R. regina & & $2 / 2$ \\
\hline 8 & Raillietnema & Chromadorea & Raillietnema sp. & Cosmocercoides & $147 / 291$ \\
\hline 9 & Phasmarhabditis & Rhabditidae & Phasmarhabditis sp. & & $53 / 135$ \\
\hline
\end{tabular}


Patterns of putative species distribution

320 For most of the host species, the sample numbers were quite low and we therefore did not

321 manage to sample all of the putative species in these hosts. PS3 was the only putative species

322 in three larger sized lemurs, brown and bamboo lemurs, whereas dogs also had PS6 (Figure

323 1). Gastropods were the only host to contain PS9.

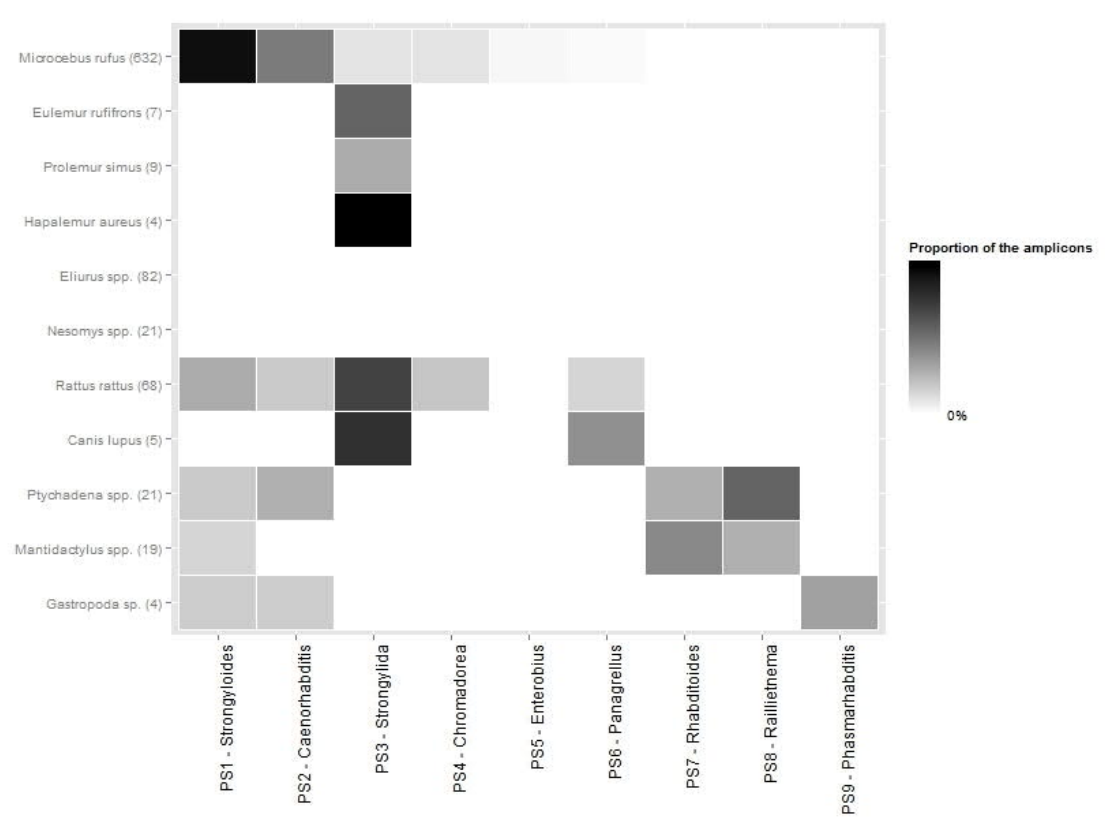

326 Figure 1: A heatmap with one host species per row and one putative species per column in

327 Ranomafana National Park, Madagascar between September 2010 and December 2012. Numbers after the

328 scientific name represents the sample size for each species. The scientific name after putative

329 parasite species number represents the lowest common ancestor of top BLAST hits from SILVA

330 database.

332 Of the taxa we sampled more than ten times, mouse lemurs, rats and frogs had nematode

333 parasites in half or more of the fecal samples (Table 1). In contrast, despite high sample

334 numbers, we found few parasites in endemic rodent species (Eliurus and Nesomys), and we 
were not successful in sequencing their parasites.

337 We used taxons with more than ten successful sequencings (mouse lemurs, black rats, frogs) 338 to explore differences between parasite assemblages. All three variables, host species (LRT= $\left.33984.54, \mathrm{p}_{\mathrm{df}=2}<0.001\right)$, sampling site $\left(\mathrm{LRT}=27.23, \mathrm{p}_{\mathrm{df}=2}=0.008\right)$ and sampling year $(\mathrm{LRT}=$ $\left.34075.27, \mathrm{p}_{\mathrm{df}=1}<0.001\right)$ had a significant effect on parasite assemblage structure. In univariate 341 tests between putative species and variables (Table 3), the differences between hosts were 342 driven by PS1 and PS2, which were less prevalent in frogs (15\% and 15\%, respectively) and 343 in rats (28\% and 20\%) than in mouse lemurs (79\% and 61\%), and PS7 and PS8, which are not 344 present in rats or mouse lemurs, but have prevalence of $32 \%$ and $42 \%$, respectively, in frogs.

345 Furthermore, Fragment site differs in several putative species (PS1-4 and PS6), whilst

346 Talatakely and Campsite sites do not significantly differ from each other for any putative

347 species. The presence of PS1 and PS2 differs significantly between years.

349 Table 3: The statistical significance of each multivariate term specified in the fitted model using 350 mvabund package from study species in Ranomafana National Park, Madagascar between September 3512010 and December 2012. The test statistic is calculated with the Wald test and p-values are calculated 352 with the PIT-trap method. The statistically significant $(\mathrm{p}<0.05)$ values are marked with bold.

\begin{tabular}{|c|c|c|c|c|c|c|c|c|c|c|c|c|}
\hline & \multicolumn{2}{|c|}{ Intercept } & \multirow{2}{*}{\multicolumn{2}{|c|}{$\begin{array}{l}\text { Host - } \\
\text { Microcebus }\end{array}$}} & \multirow{2}{*}{\multicolumn{2}{|c|}{ Host - Frogs }} & \multirow{2}{*}{\multicolumn{2}{|c|}{$\begin{array}{l}\text { Site - } \\
\text { Fragment }\end{array}$}} & \multirow{2}{*}{\multicolumn{2}{|c|}{$\begin{array}{l}\text { Site - } \\
\text { Talatakely }\end{array}$}} & \multirow{2}{*}{\multicolumn{2}{|c|}{ Year }} \\
\hline & \multirow[b]{2}{*}{ Wald } & \multirow[b]{2}{*}{$\mathrm{p}$} & & & & & & & & & & \\
\hline & & & Wald & $\mathrm{p}$ & Wald & $\mathrm{p}$ & Wald & $\mathrm{p}$ & Wald & $\mathrm{p}$ & Wald & $\mathrm{p}$ \\
\hline PS1 & 3.29 & $<0.001$ & 3.631 & 0.004 & 1.353 & 0.12 & 0.038 & 0.01 & 0.802 & 0.93 & 3.282 & 0.007 \\
\hline PS2 & 6.04 & $<0.001$ & 1.170 & 0.38 & 1.52 & 0.12 & 0.037 & 0.01 & 0.454 & 0.93 & 6.037 & $<0.001$ \\
\hline PS3 & 0.70 & 0.59 & 5.563 & $<0.001$ & 0.077 & 0.12 & 2.518 & 0.007 & 1.557 & 0.48 & 0.699 & 0.59 \\
\hline PS4 & 2.85 & 0.012 & 0.076 & 0.60 & 0.069 & 0.12 & 2.069 & 0.01 & 0.161 & 0.93 & 2.848 & 0.01 \\
\hline
\end{tabular}




\begin{tabular}{lllllllllllllll}
\hline PS5 & 1.43 & 0.29 & 0.064 & 0.60 & 0.002 & 0.34 & 0 & 0.66 & 0.737 & 0.93 & 1.433 & 0.29 & \\
PS6 & 0.86 & 0.59 & 1.157 & 0.37 & 0.061 & 0.12 & $\mathbf{1 . 8 9 9}$ & $\mathbf{0 . 0 1}$ & 0.325 & 0.93 & 0.865 & 0.59 & \\
PS7 & 0.00 & 0.59 & $\mathbf{0 . 0 0 0}$ & $\mathbf{0 . 6 1}$ & 0.073 & 0.12 & 0 & 0.66 & 0 & 0.93 & 0 & 0.59 & \\
PS8 & 0.00 & 0.59 & $\mathbf{0 . 0 0}$ & $\mathbf{0 . 6 1}$ & 0.075 & 0.34 & 0 & 0.66 & 0 & 0.91 & 0 & 0.59 &
\end{tabular}

\section{Discussion}

355 Our results show that metabarcoding can be used to non-invasively resolve the diversity in

356 previously uninvestigated partial parasite assemblages. Non-invasive sampling and

357 metabarcoding revealed differing parasite assemblages in sympatric species inhabiting the

358 Malagasy rainforest. Nevertheless, the detection of the parasite sharing between different

359 species was limited by the trade-offs inherent in the choice of the marker gene and sampling

360 method.

362 We found statistically significant differences in parasite occurrence between host species,

363 between years and between sampling localities (Table 3). While campsite and Talatakely were

364 highly similar in parasite occurrence, more distantly situated forest fragments differed in the

365 occurrence of PS1, PS2 and PS4. Parasite assemblage in black rats did not differ from frogs,

366 but they did have significant difference to mouse lemurs (Table 3; Figure 1) This is mostly

367 driven by a difference of degree, not difference of kind, as the host species have similar

368 putative species. The mouse lemurs and black rats, the two most extensively sampled host

369 species, seem to host almost identical groups of putative species, with the exception of PS5

370 (matched to Enterobius) which appeared exclusively, though rarely, in mouse lemurs (Figure

371 1). Nevertheless, as the resolution of the marker gene is limited, we do not know whether

372 putative species contain one or more parasite species. That is, we do not know whether mouse

373 lemurs and rats share parasite species or if the number of putative species is representative of 
374 their actual parasite richness. In contrast, frogs were differentiated by the presence of frog-

375 specific putative species. As the lowest common ancestor would suggest, these putative

376 species belong to taxa previously known to infect amphibians and gastropods. While we

377 sampled the endemic rodents well (Eliurus spp. and Nesomys spp.), we rarely detected

378 parasites in the feces (Table 1), which means they probably have parasite species not detected

379 by our method. While we are unable to identify all black rat parasites, their parasite

380 communities differ, at least partially, compared to the endemic rodents. This result is in line

381 with previous studies on the ectoparasites of endemic rodents and black rats, which showed

382 that endemic rodents did not have any invasive fleas while they were abundant on black rats,

383 especially on disturbed sites (Laakkonen et al. 2003).

385 In assessing the usefulness of parasite identification methods, whether it is a new

386 metabarcoding method or traditional coproscopy, there are three distinct questions: i) how

387 well methods detect parasite species, ii) how they resolve the number of parasitic taxa and iii)

388 how accurate is the identification of these species. Mitochondrial cytochrome oxidase subunit

389 I (COI) gene is the standard marker gene for barcoding metazoan species (Hebert et al. 2003),

390 but it has proved impractical for nematodes. We used the ribosomal small subunit gene (18S)

391 as the barcode for nematodes for several reasons: 18S has conserved primer sites across all

392 nematodes, amplicons can be used for identification (Porazinska et al. 2009, Tanaka et al.,

393 2014) and it is the most sequenced gene region in nematodes. As this gene region is relatively

394 conserved it underestimates species richness (De Ley et al. 2005; Tang et al. 2012).

395 Nevertheless, for mouse lemur putative species richness, we are comparable with with

396 previous studies (Raharivololona and Ganzhorn 2010; Raharivololona and Ganzhorn 2009)

397 and our previous study suggests we sampled mouse lemurs exhaustively (Aivelo et al. 2015). 
The only detected putative species, PS3, in medium-sized lemurs (Eulemur, Hapalemur, Prolemur) is compatible with previous surveys in Ranomafana which found identical "strongylid" eggs in several medium-sized lemurs (Hogg 2003, as cited in Irwin and Raharison (2009)). The number of putative species in black rats is comparable to previous

402 studies of rodents in Ranomafana National Park (Lehtonen, unpubl.): PS1 and PS3 match 403 previously detected Strongyloides ratti and Nippostrongylus brasiliensis. We found, however, 404 fewer species than Raharivololona et al. (2007) did in Mandena where they identified 15 405 morphospecies across 36 samples. To assess the match between coproscopy and sequencing, 406 we dissected black rats and morphologically identified their parasites as Nippostrongylus sp. 407 and Mastophorus sp. Nippostrongylus sp. positive rats had PS3 also in the larval samples, 408 though one of the larval samples yielded a different identification, PS1. To get a conservative estimate of species richness, we excluded any OTUs that were identified as soil nematodes and were exclusively found in samples known to have come in contact with the cage floor. There were two species (PS2: Caenorhabditis, PS6: Panagrellus), which had their closest 412 match to soil nematodes but which were also present in the samples which were not in contact 413 with the soil or trap floors, i.e., samples collected directly from defecating animals.

414 Furthermore, Baermann's method only allows for detection of living nematodes, which rules 415 out nematode detection through geophagy or other accidental ingestion, which means that the 416 possibility of these species being free-living is small. As these putative species were 417 encountered in several species, it is possible that they are composed of several actual species, 418 some of which are soil nematodes. In the future, expanded genetic databases could resolve, 419 which species these putative species actually belong to.

421 Baermann's method is inherently limiting with respect to nematode communities resolution as 
not all nematode parasite species have free-living larval forms. For example, we were unable to detect Mastophorus sp., a large-sized nematode that inhabits the stomach of rodents, in the larval samples. Indeed, Mastophorus does not have free-living larvae and could not be isolated by Baermann's method. Endemic rodents can also carry Mastophorus as an earlier survey found it in both endemic rodent genera (Jukka T. Lehtonen, unpubl.). Also Enterobius should not be detected by Baermann's method as eggs are infectious without a free-living larval stage. We suspect that the low prevalence of Enterobius represents chance amplifications of Enterobius genetic material and thus underestimates the total prevalence. An

430 alternative method would be to isolate parasite DNA from the feces as in Tanaka et al. (2014),

431 but this in turn could lead to difficulties distinguishing actual parasites inhabiting the

432 gastrointestinal tract and accidentally ingested parasites, for example, from the diet.

433 Irrespective, of what fecal analysis methods is used, they can only detect helminths when they 434 are laying eggs.

436 The specificity of assigned labels varied depending on the nematode clade. For example, the 437 only Enterobius sequence in the SILVA database would form its own cluster, i.e., it can be 438 distinguished from all the other nematode species in the database (Table 2). In comparison, 439 clusters based on nematode sequences from Rhabditidae or Chromadorea can contain several 440 different species. It should be noted that this is predominantly a problem for labelling these 441 clusters: within these taxa, there can be a high number of different clusters, i.e., they can be 442 differentiated from each other, but they are still labelled as Rhabditidae and Chromadorea. 443 Although there are almost 19,000 18S sequences in Genbank, from over 4,600 different 444 species of nematode (as of February 2017, excluding environmental and metagenomic data), 445 our samples rarely got perfect matches (Table 2). This is unsurprising as there are very few 
sequences for intestinal nematodes from Malagasy animals published. It is also probable that our analyses contain species that have not been previously described. Nevertheless, the lowest common taxonomical ancestor of top scoring BLAST hits is a valid and practical way of labeling putative species. Obviously, future work will be required to determine the exact relationships between the nematode OTUs shared by the endemic and non-endemic hosts.

This study also demonstrates the challenge of choosing the target region for a barcoding analysis: PCR amplification with universal primers requires regions with high sequence conservation whereas high overall conservation limits resolution for identification on lower taxonomic levels (Powers et al. 2011). The primers with a more informative target region or longer amplicons could enhance the resolution of the method. One concern for the metabarcoding approach is that the success of amplification and sequencing was quite low (Table 1). This could be due to low levels of DNA, the nematode cuticle or the presence of inhibiting substances in fecal samples. We do not believe the low success rate is due to our

460 primers systematically failing to amplify some nematode species as the success rate for the 461 second attempt of isolation and amplification for failed samples was comparable to the first 462 (30\% vs. $22 \%$ ). This low success rate, though, implies that using fecal parasite DNA, i.e., larvae or eggs, for DNA isolation could pose additional challenges for metabarcoding parasite 464 communities.

In conclusion, metabarcoding is a promising approach for non-invasive survey of intestinal

467 parasites. Nevertheless, our approach was limited by Baermann's method and low resolution 468 of the $18 \mathrm{~S}$ marker gene. There is also a need for more robust DNA isolation methods to 469 ensure successful amplification. Further development could make this a useful tool for 
470 assessing parasite communities more holistically in threatened host communities. Our results 471 show that well-sampled host species had differing parasite assemblages and both sampling 472 site and year affected parasite assemblages. Though there was an overlap of putative species 473 in sympatric host species, we cannot conclude whether these are same or different parasite 474 species. 


\section{References}

Aivelo, T., \& Medlar, A. (2017). Opportunities and challenges in metabarcoding approaches for helminth community identification in wild mammals. Parasitology. doi: $10.1017 / \mathrm{S} 0031182017000610$

Aivelo, T., Medlar, A., Löytynoja, A., Laakkonen, J., \& Jernvall, J. (2015). Tracking year-toyear changes in intestinal nematode communities of rufous mouse lemurs (Microcebus rufus). Parasitology, 142(8), 1095-1107. doi:10.1017/S0031182015000438

Andriaholinirina, N., Baden, A., Blanco, M., Chikhi, L., Cooke, A., Davies, N., et al. (2014a). Hapalemur aureus. The IUCN Red List of Threatened Species, 2014, e.T9672A16119513. doi:10.2305/IUCN.UK.2014-1.RLTS.T9672A16119513.en

Andriaholinirina, N., Baden, A., Blanco, M., Chikhi, L., Cooke, A., Davies, N., et al. (2014b). Prolemur simus. The IUCN Red List of Threatened Species, 2014, e.T9674A16121559. doi:10.2305/IUCN.UK.2014-1.RLTS.T9674A16121559.en

Avramenko, R. W., Redman, E. M., Lewis, R., Yazwinski, T. A., Wasmuth, J. D., \& Gilleard, J. S. (2015). Exploring the gastrointestinal "nemabiome": Deep amplicon sequencing to quantify the species composition of parasitic nematode communities. PLoS ONE, 10(12), 1-18. doi:10.1371/journal.pone.0143559

Baermann, G. (1917). Eine einfache methode zur auffindung von Ankylostomum (Nematoden) larven in erdproben. Tijdschrid Ergeneeskd, 57, 131-137.

Bhadury, P., \& Austen, M. C. (2010). Barcoding marine nematodes : an improved set of nematode 18S rRNA primers to overcome eukaryotic co-interference. Hydrobiologia, 
Bik, H. M., Porazinska, D. L., Creer, S., Caporaso, J. G., Knight, R., \& Thomas, W. K. (2012). Sequencing our way towards understanding global eukaryotic biodiversity. Trends in Ecology \& Evolution, 27(4), 233-43. doi:10.1016/j.tree.2011.11.010

Blaxter, M., Mann, J., Chapman, T., Thomas, F., Whitton, C., Floyd, R., \& Abebe, E. (2005). Defining operational taxonomic units using DNA barcode data. Philosophical Transactions of the Royal Society B: Biological Sciences, 360(1462), 1935-43. doi:10.1098/rstb.2005.1725

Camacho, C., Coulouris, G., Avagyan, V., Ma, N., Papadopoulos, J., Bealer, K., \& Madden, T. L. (2009). BLAST+: architecture and applications. BMC bioinformatics, 10, 421. doi:10.1186/1471-2105-10-421

Chakraborty, D., Hussain, S., Reddy, D. M., Raut, S., Tiwari, S., Kumar, V., \& Umapathy, G. (2015). Mammalian gastrointestinal parasites in rainforest remnants of Anamalai Hills, Western Ghats, India. Journal of Biosciences, 40(2), 399-406. doi:10.1007/s12038-0159517-5

Dallas, T., \& Presley, S. J. (2014). Relative importance of host environment, transmission potential and host phylogeny to the structure of parasite metacommunities. Oikos, 123(7), 866-874. doi:10.1111/oik.00707

De Ley, P., De Ley, I. T., Morris, K., Abebe, E., Mundo-Ocampo, M., Yoder, M., et al. (2005). An integrated approach to fast and informative morphological vouchering of nematodes for applications in molecular barcoding. Philosophical Transactions of the Royal Society of London B: Biological sciences, 360(1462), 1945-58. doi:10.1098/rstb.2005.1726 
Dobson, A. P., \& May, R. M. (1986). Patterns of invasions by pathogens and parasites. In H. A. Mooney \& J. A. Drake (Eds.), Ecology of Biological Invasions of North America and Hawaii (pp. 58-76). New York, USA: Springer-Verlag.

Dunn, A. M., Torchin, M. E., Hatcher, M. J., Kotanen, P. M., Blumenthal, D. M., Byers, J. E., et al. (2012). Indirect effects of parasites in invasions. Functional Ecology, 26(6), 12621274. doi:10.1111/j.1365-2435.2012.02041.x

Edgar, R. C., Haas, B. J., Clemente, J. C., Quince, C., \& Knight, R. (2011). UCHIME improves sensitivity and speed of chimera detection. Bioinformatics, 27(16), 2194-2200. doi:10.1093/bioinformatics/btr381

Freeland, W. J. (1983). Parasites and coexistence of animal host species. The American Naturalist, 121(2), 223-236.

Gillespie, T. R. (2006). Noninvasive assessment of gastrointestinal parasite infections in freeranging primates. International Journal of Primatology, 27(4), 1129-1143. doi:10.1007/s10764-006-9064-X

Hudson, P., \& Greenman, J. (1998). Competition mediated by parasites: biological and theoretical progress. Trends in Ecology \& Evolution, 13(10), 387-390.

Irwin, M. T., \& Raharison, J. L. (2009). A review of the endoparasites of the lemurs of Madagascar. Malagasy Nature, 2, 66-93.

Kelly, D. W., Paterson, R. A., Townsend, C. R., Poulin, R., \& Tompkins, D. M. (2009). Parasite spillback: a neglected concept in invasion ecology? Ecology, 90(8), 2047-2056.

Kouassi, R. Y. W., McGraw, S. W., Yao, P. K., Abou-Bacar, A., Brunet, J., Pesson, B., et al. 

(2015). Diversity and prevalence of gastrointestinal parasites in seven non-human primates of the Taï National Park, Côte d'Ivoire. Parasite, 22, 1. doi:10.1051/parasite/2015001

Laakkonen, J., Goodman, S. M., Duchemin, J. B., \& Duplantier, J. M. (2003). Trypomastigotes and potential flea vectors of the endemic rodents and the introduced Rattus rattus in the rainforests of Madagascar. Biodiversity and Conservation, 12(8), 1775-1783. doi:10.1023/A:1023611727357

Lott, M. J., Hose, G. C., \& Power, M. L. (2015). Parasitic nematode communities of the red kangaroo, Macropus rufus: richness and structuring in captive systems. Parasitology Research, (114), 2925-2932. doi:10.1007/s00436-015-4494-z

Loudon, J. E., \& Sauther, M. L. (2013). Verreaux's sifaka (Propithecus verreauxi ) and ringtailed lemur (Lemur catta ) endoparasitism at the Bezà Mahafaly Special Reserve. Madagascar Conservation \& Development, 8(1), 21-28.

MacLeod, C. J., Paterson, A. M., Tompkins, D. M., \& Duncan, R. P. (2010). Parasites lost - do invaders miss the boat or drown on arrival? Ecology Letters, 13(4), 516-27. doi:10.1111/j.1461-0248.2010.01446.x

Maldonado-López, S., Maldonado-López, Y., Gómez-Tagle Ch, A., Cuevas-Reyes, P., \& Stoner, K. E. (2014). Patterns of infection by intestinal parasites in sympatric howler monkey (Alouatta palliata) and spider monkey (Ateles geoffroyi) populations in a tropical dry forest in Costa Rica. Primates, 55(3), 383-92. doi:10.1007/s10329-0140413-7

561 Medlar, A., Aivelo, T., \& Löytynoja, A. (2014). Séance : reference-based phylogenetic 
analysis for 18S rRNA studies. BMC Evolutionary Biology, 14(235). doi:10.1186/s12862-014-0235-7

Muriuki, S. M. K., Murugu, R. K., Munene, E., Karere, G. M., \& Chai, D. C. (1998). Some gastro-intestinal parasites of zoonotic ( public health ) importance commonly observed in old world non-human primates in Kenya. Acta Tropica, 71, 73-82.

Nowak, R. M. (1999a). Walker's Mammals of the World, Volume I (6th ed.). Baltimore and London: The John Hopkins University Press.

Nowak, R. M. (1999b). Walker's Mammals of the World, Volume II (6th ed.). Baltimore and London: The John Hopkins University Press.

Petrášová, J., Modrý, D., Huffman, M. a., Mapua, M. I., Bobáková, L., Mazoch, V., et al. (2010). Gastrointestinal Parasites of Indigenous and Introduced Primate Species of Rubondo Island National Park, Tanzania. International Journal of Primatology, 31(5), 920-936. doi:10.1007/s10764-010-9439-х

Porazinska, D. L., Giblin-Davis, R. M., Sung, W., \& Thomas, W. K. (2010). Linking operational clustered taxonomic unitus (OCTUs) from parallel ultra sequencing (PUS) to nematode species. Zootaxa, 2427, 55-63.

Pourrut, X., Diffo, J. L. D., Somo, R. M., Bilong Bilong, C. F., Delaporte, E., LeBreton, M., \& Gonzalez, J. P. (2011). Prevalence of gastrointestinal parasites in primate bushmeat and pets in Cameroon. Veterinary Parasitology, 175(1-2), 187-91. doi:10.1016/j.vetpar.2010.09.023

Powers, T., Harris, T., Higgins, R., Mullin, P., Sutton, L., \& Powers, K. (2011). MOTUs, morphology, and biodiversity estimation: a case study using nematodes of the suborder 

48. http://www.pubmedcentral.nih.gov/articlerender.fcgi?artid=3380479\&tool=pmcentrez\&r endertype $=$ abstract

Quast, C., Pruesse, E., Yilmaz, P., Gerken, J., Schweer, T., Yarza, P., et al. (2013). The SILVA

Quince, C., Lanzen, A., Davenport, R. J., \& Turnbaugh, P. J. (2011). Removing noise from pyrosequenced amplicons. BMC Bioinformatics, 12(1), 38. doi:10.1186/1471-2105-12-

Raharivololona, B., \& Ganzhorn, J. (2010). Seasonal variations in gastrointestinal parasites excreted by the gray mouse lemur Microcebus murinus in Madagascar. Endangered Species Research, 11, 113-122. doi:10.3354/esr00255

Raharivololona, B. M., \& Ganzhorn, J. U. (2009). Gastrointestinal parasite infection of the gray mouse lemur (Microcebus murinus) in the littoral forest of Mandena, Madagascar: effects of forest fragmentation and degradation. Madagascar Conservation \& Development, 4(2), 103-112.

Raharivololona, B. M., Rakotondravao, \& Ganzhorn, J. U. (2007). Gastrointestinal Parasites of Small Mammals in the Littoral Forest of Mandena. In J. U. Ganzhorn, S. M. Goodman, \& M. Vincelette (Eds.), Biodiversity, Ecology, and Conservation of Littoral Ecosystems in the Region of Tolagnaro (Fort Dauphin), Southeastern Madagascar. (pp. 247-258). Washington, D.C.: Smithsonian Institution. 
606 Schwensow, N., Dausmann, K., Eberle, M., Fietz, J., \& Sommer, S. (2010). Functional

607

608

609

610

611

612

613

614

615

616

617

618

619

620

621

622

623

624

625

626

627 associations of similar MHC alleles and shared parasite species in two sympatric lemurs. Infection, Genetics and Evolution, 10(5), 662-8. doi:10.1016/j.meegid.2010.03.012

Smith, J. A., \& Kinsella, J. M. (2011). Gastric spiruridiasis caused by Mastophorus muris in a captive population of striped possums (Dactylopsila trivirgata). Journal of Zoo and Wildlife Medicine, 42(2), 357-359.

Stamatakis, A. (2006). RAxML-VI-HPC: maximum likelihood-based phylogenetic analyses with thousands of taxa and mixed models. Bioinformatics, 22(21), 2688-90. doi:10.1093/bioinformatics/btl446

Taberlet, P., Coissac, E., Pompanon, F., Brochmann, C., \& Willerslev, E. (2012). Towards next-generation biodiversity assessment using DNA metabarcoding. Molecular Ecology, 33, 2045-2050. http://onlinelibrary.wiley.com/doi/10.1111/j.1365294X.2012.05470.x/full\%5Cnpapers2://publication/uuid/6969E8B6-A569-4084-8966F9A2E26F635E

Tanaka, R., Hino, A., Tsai, I. J., Palomares-Rius, J. E., Yoshida, A., Ogura, Y., et al. (2014). Assessment of helminth biodiversity in wild rats using 18S rDNA based metagenomics. PloS one, 9(10), e110769. doi:10.1371/journal.pone.0110769

Tang, C. Q., Leasi, F., Obertegger, U., Kieneke, A., Barraclough, T. G., \& Fontaneto, D. (2012). The widely used small subunit $18 \mathrm{~S}$ rDNA molecule greatly underestimates true diversity in biodiversity surveys of the meiofauna. Proceedings of the National Academy of Sciences of the United States of America, 109(40), 16208-12. doi:10.1073/pnas.1209160109 
628 Taraschewski, H. (2006). Hosts and parasites as aliens. Journal of Helminthology, 80(2), 99128. doi:10.1079/JOH2006364

630 Team, R. C. (2013). R: A language and environment for statistical computing. Vienna, Austria.: R Foundation for Statistical Computing. http://www.r-project.org/

632

634

635

Teichroeb, J. a, Kutz, S. J., Parkar, U., Thompson, R. C. A., \& Sicotte, P. (2009). Ecology of the gastrointestinal parasites of Colobus vellerosus at Boabeng-Fiema, Ghana: possible anthropozoonotic transmission. American Journal of Physical Anthropology, 140(3), 498-507. doi:10.1002/ajpa.21098

Torchin, M. E., Lafferty, K. D., Dobson, A. P., McKenzie, V. J., \& Kuris, A. M. (2003). Introduced species and their missing parasites. Nature, 421, 628-630. doi:10.1038/nature01346.1.

Trejo-Macías, G., \& Estrada, A. (2012). Risk factors connected to gastrointestinal parasites in mantled Alouatta palliata mexicana and black howler monkeys Alouatta pigra living in continuous and in fragmented rainforests in Mexico. Current Zoology, 58(3), 375-383.

Trejo-Macías, G., Estrada, A., \& Mosqueda Cabrera, M. Á. (2007). Survey of helminth parasites in populations of Alouatta palliata mexicana and A. pigra in continuous and in fragmented habitat in Southern Mexico. International Journal of Primatology, 28(4), 931-945. doi:10.1007/s10764-007-9137-5

Wang, Y., Naumann, U., Wright, S., Eddelbuettel, D., \& Warton, D. I. (2016). mvabund: Statistical Method for Analysing Multivariate Abundance Data. http://cran.rproject.org/package $=$ mvabund

Wimmer, B., Craig, B. H., Pilkington, J. G., \& Pemberton, J. M. (2004). Non-invasive 

assessment of parasitic nematode species diversity in wild Soay sheep using molecular markers. International Journal for Parasitology, 34, 625-631.

652 doi:10.1016/j.ijpara.2003.11.022

653 Wright, P. C., \& Andriamihaja, B. (2002). Making a rain forest national park work in 654 Madagascar: Ranomfana National Park and its long-term research commitment. In J. 655 Terborgh, C. van Schaik, L. Davenport, \& M. Rao (Eds.), Making parks work: strategies 656 for preserving tropical nature (pp. 112-136). Washington DC, USA: Island Press. 